\title{
Distributed Opportunistic Scheduling in IEEE 802.11 WLANs
}

\author{
Seong-il Hahm ${ }^{1}$, Jongwon Lee ${ }^{2}$, and Chong-kwon Kim ${ }^{1}$ \\ ${ }^{1}$ School of Electrical Engineering and Computer Science \\ Seoul National University, Seoul, 151-742, Republic of Korea \\ \{siham, ckim\}@popeye.snu.ac.kr \\ 2 School of Computer Science \& Electrical Engineering \\ Handong Global University, Pohang, 791-708, Republic of Korea \\ ljw@handong.edu
}

\begin{abstract}
Opportunistic scheduling monitors the receivers' channel states and schedules packets to the receivers in relatively good channel conditions. Opportunistic scheduling can be easily implemented in cellular networks such as the 1 xEVDO system because the channel state report function is embedded in the system. To apply opportunistic scheduling to WLANs, deficient of channel report functions, we first devise efficient channel probing mechanisms. Several opportunistic scheduling methods for WLANs have been proposed recently. These previous methods limit the candidate receivers and may not fully realize the potential multiuser diversity gains. In this paper, we develop new opportunistic scheduling called WDOS (Wireless LAN Distributed Opportunistic Scheduling). That is based on a modified RTS/CTS exchange scheme. In WDOS, a sender broadcasts a BRTS (Broadcast RTS) to all receivers. A receiver responds with a CTS after a backoff delay. The value of the backoff delay is determined such that the receivers in relatively better channel conditions acquire channel accesses. We evaluate the performance of WDOS both via an analytic method and via computer simulations. Our performance study shows that WDOS achieves the performance near optimal.
\end{abstract}

Keywords: WLANs, Opportunistic scheduling, Multiuser diversity, Distributed scheduling, Channel probing, Temporal fairness.

\section{Introduction}

Dynamic fluctuations of channel quality in wireless networks provide the opportunities to improve the performance of the systems. Channel fluctuations occur both in a short-term scale (small-scale fading) and in a long-term scale (largescale propagation) [11]. In this paper, we focus on small-scale fading such as Rayleigh and Ricean fading because they are commonly used to describe the flat fading channel characteristics in the outdoor and indoor environment, respectively. Suppose that a sender has packets to send to several receivers. If the sender knows the receivers' channel conditions, it transmits packets to the 
receiver in the best channel condition at a high data rate. Because receivers in good channel conditions get service, the overall performance of the system improves. The performance gains obtained by exploiting the dynamic fluctuation of channel quality are called multiuser diversity gains [7] or scheduling gains.

Opportunistic scheduling algorithms, which exploit the multiuser diversity, need to know all receivers' channel states. In cellular networks such as 1xEVDO [10], mobile stations (i.e. handset) report their channel qualities to a Base Station (BS) periodically. Based on the reported channel conditions, the BS schedules a packet to the most suitable receiver.

To apply opportunistic scheduling to WLANs, deficient of channel report functions, we first devise efficient channel probing mechanisms. Recently, Medium Access Diversity (MAD) [6] and Opportunistic packet Scheduling and Media Access control (OSMA) [12] have been proposed to exploit multiuser diversity in WLANs. To explicitly probe receivers' channel qualities, both MAD and OSMA use the modified RTS/CTS exchange mechanism. To confine the overhead of channel probing, both methods limit the channel probing to three or four candidates. MAD and OSMA may not be able to fully realize potential multiuser diversity gains because they exclude some receivers in scheduling.

In this paper, we propose a new WLAN opportunistic scheduling algorithm called WLAN Distributed Opportunistic Scheduling (WDOS). Like MAD and OSMA, WDOS also uses a modified RTS and CTS exchange to probe channel conditions. However, WDOS invites all receivers to report their channel conditions without excessive probing overheads and has a potential to fully realize multiuser diversity gains.

A brief description of WDOS is as follows. A sender broadcasts a channel probing message called BRTS (Broadcast RTS) to all receivers. Receiving the probing message, each receiver waits a random backoff period before responding with a CTS frame. The station that has the shortest backoff period will transmit a CTS message first. Hearing the first CTS message, other receivers give up their CTS transmissions. The sender transmits a packet to the station that transmits the CTS message. The backoff period of each receiver is determined by its relative instantaneous channel quality. A station in a better relative channel condition has a shorter backoff delay and has a better chance to be scheduled. By using relative channel quality, not absolute one, WDOS can guarantee temporal fairness even though the average channel qualities of receivers are different.

We have evaluated the performance of WDOS using both an analytical method and computer simulations. The performance results indicate that the throughput of WDOS is $30 \%$ higher than that of MAD and OSMA if there are more than ten receivers. The advantage of WDOS becomes more significant as the number of receivers increases. Given that the channel qualities of all receivers are i.i.d. with the same average, the throughput of WDOS approaches to that of the max C/I scheduler [8] and achieves the throughput near maximal. In the case that the average channel qualities of receivers are different, WDOS provides temporal fairness like proportional fair scheduling [5]. 
The rest of the paper is organized as follows. Section 2 describes several previous results related to our work. Section 3 illustrates the basic framework of WDOS and explains a possible implementation scheme. In Section 4, we provide an analytic model for the performance analysis of WDOS. Section 5 evaluates the performance of WDOS and compares its performance with other opportunistic scheduling mechanisms using both the analytic model and computer simulations. The conclusions are given in Section 6 .

\section{Related Works}

Cellular network systems such as 1xEVDO have an intrinsic channel information report mechanism. But the IEEE 802.11 WLANs do not support the mechanism. Therefore, it is more difficult to adopt opportunistic scheduling in WLANs than cellular network systems.

In spite of the difficulty, two opportunistic scheduling algorithms for WLANs have been proposed recently: MAD [6] and OSMA [12]. Both schemes modify the RTS/CTS exchange to probe channel conditions of selected candidate receivers. In MAD, a sender selects a few candidate receivers and probes the channel states of the candidates by sending a modified RTS frame. MAD explicitly specifies the selected candidates by recording their addresses in the RTS frame. To confine the overhead of channel probing, MAD limits the number of candidates to three. Each candidate receiver responds with a CTS frame specifying its instantaneous channel quality. To avoid CTS response collisions, the receivers transmit CTS frames according to the order that the sender specifies in the RTS frame. Based on the reported channel information, the sender selects a receiver.

OSMA is another opportunistic scheduling method designed for WLANs. Like MAD, a sender selects candidate receivers and transmits a channel probing message to the candidates. OSMA limits the number of candidate receivers to four. When a candidate receiver hears the probing message from a sender, it measures its channel quality and tests if the channel quality is better than a certain threshold. The receiver responds to the probe message only if its channel quality is better than the threshold. As soon as the first response is detected, the probing process terminates and the sender transmits data frames to the receiver that sends the response. OSMA uses a CTS collision avoidance mechanism similar to MAD's.

\section{Proposed Scheme}

\subsection{Framework}

Our work begins from the question, "How can we fully realize multiuser diversity gains without excessive overheads of channel probing?" MAD and OSMA fail to fully realize multiuser diversity gains because they limit the number of candidate receivers. Because the probing overheads of MAD and OSMA increase in 


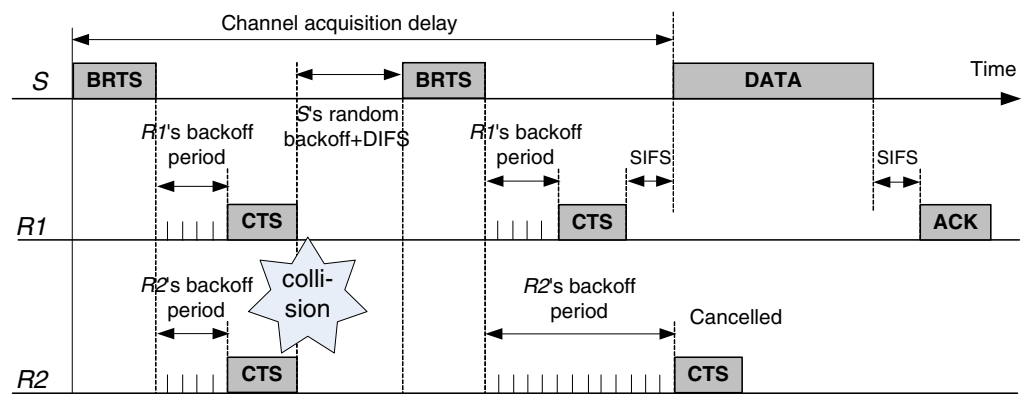

Fig. 1. Channel probing procedure of WDOS

proportion to the number of candidates, these methods cannot expand the number of candidates. Both methods should optimize a trade-off relation between the multiuser diversity gain and the probing overhead. It has been shown that the numbers three and four optimize the trade-off relation in MAD and OSMA, respectively $[6],[12]$.

The essence of opportunistic scheduling for WLANs is the channel probing mechanism. In order to fully achieve multiuser diversity gains, we have to probe all receivers without excessive probing overheads. To break the trade-off relation, we propose a contention-based probing scheme. The scheme, named WDOS, employs the RTS/CTS handshake mechanism with some modifications. A sender broadcasts a Broadcast RTS (BRTS) control frame to all the active receiver:1. Each receiver waits for a time called the backoff period before responding with a CTS control frame. There are the chances that two receivers randomly select the same backoff period and CTS frames collide. Collide or not, the first CTS finishes the current channel probing period. Fig. 1 briefly shows the channel probing procedure of WDOS with a simple example. The sender, $S$, probes the channel conditions of two active receivers, $R 1$ and $R 2$, by broadcasting a BRTS frame. $R 1$ and $R 2$ determine their backoff periods based on estimated channel quality. At first, they have the same backoff time and their CTS frames are collided. After waiting for a binary exponential backoff period, the sender retransmits a BRTS frame. At this time, $R 1$ has a shorter backoff period than $R 2$, and $R 1$ gains the channel. We define the channel acquisition delay as the time from the first BRTS transmission to the beginning of DATA transmission. If a CTS collision occurs, the acquisition delay becomes longer.

\subsection{Backoff Period}

The performance of WDOS depends on the effectiveness and efficiency of the mechanism that selects the CTS backoff periods. A good backoff mechanism must satisfy two requirements; maximum multiuser diversity gains and minimum overheads of channel probing. In addition, we pursue the third requirement, fairness of scheduling. The first and third requirements are easy to accomplish; the

\footnotetext{
${ }^{1}$ A station is called an active receiver to which a sender has pending data frames.
} 


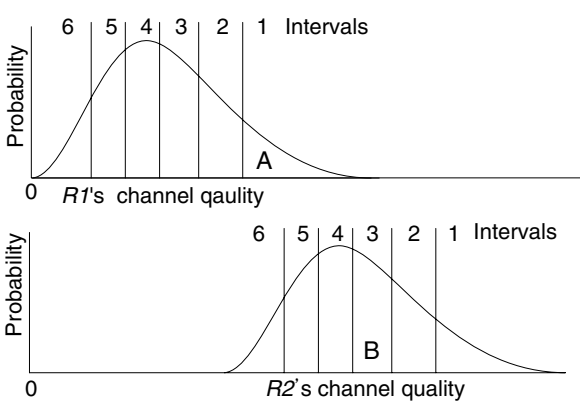

Fig. 2. Channel quality distribution and backoff mechanism

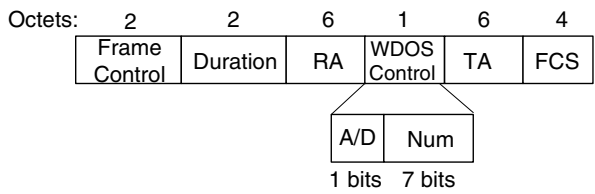

(a) BRTS

Octets:
\begin{tabular}{c|c|c|c|c|c|}
\hline $\begin{array}{c}\text { Frame } \\
\text { Control }\end{array}$ & Duration & RA & R & TA & FCS \\
\hline
\end{tabular}

(b) CTS

Fig. 3. WDOS control frame formats

first by assigning shorter backoff delays to receivers in better channel qualities, and the third by using relative, not absolute, channel qualities.

Let us elaborate the backoff mechanism with an example. For the sake of simplicity, we suppose that two receivers' channel quality follows the identical distribution with different averages. Each p.d.f. is divided into a fixed number of intervals of equal probability as shown in Fig. 2. If a receiver is in interval 1, it can respond to the BRTS frame without backoff. In interval 2, it should wait one slot, and so on. Suppose $R 1$ and $R 2$ are in the first interval (denoted by 'A') and in the third interval (denoted by 'B'), respectively. Even though absolute channel quality of $R 2$ is better than that of $R 1, R 1$ grasps the channel because its relative channel quality is better than $R 2$ 's.

The number of partitions, $L$, is an important parameter that ultimates the efficiency of the channel probing mechanism. Large values of $L$ reduce the chance of collisions but delay the first CTS response. Small values of $L$ exert the exactly opposite effects on the collision probability and the responsiveness. Apparently, the optimal values of $L$ increase in proportion to the number of receivers, $N$. The question is "Is the relation linear?". Our performance study indicates that the relationship is practically linear. Section 5 reveals the relationship in a greater detail. Another concern is the continual collisions; two or more receivers in the same relative channel position collide in each of consecutive channel probing periods if their relative channel positions persist. There are several methods that solve the problem. One is to add a random factor to the backoff delay and another is the binary exponential backoff mechanism. For simplicity, WDOS adopts the former method. Let $M$ be the randomization parameter. The backoff delay of a receiver in the $i^{t h}$ interval is determined as $((i-1) * M+\operatorname{rand}[0, \cdots, M-1]) *$ SlotTime, where SlotTime is one backoff slot time.

WDOS supplies a fail-safe mechanism against abnormal operations. One abnormal condition is the failure of BRTS frame transmission due to collisions or sudden channel degradations. To prevent the sender from waiting for CTS for a prolonged time, WDOS specifies a parameter $B_{\max }$ which is the maximum number of slots before the sender stops the current probing period and 
starts a new one. We can set $B_{\max }$ to a value much smaller than the theoretical limit, $L * M-1$, because the probability that all $N$ receivers are in the poorest channel condition is very small. Moreover, we can bypass the data transmission opportunities in poor channel condition by adopting small $B_{\max }$.

Another factor that we should consider is the hidden terminal problem. The delayed CTS mechanisms [6][12] may suffer from an additional hidden terminal problem. A CTS frame delayed more than EIFS $-2 * S I F S-T_{C T S}$ could induce collisions, where $T_{C T S}$ is the CTS transmission time. The hidden terminal problem does not occur in the infrastructure mode operation, the main application target of WDOS. Even in the ad-hoc mode, the hidden terminal problem is scarcely observed in our performance study. Due to the limited space, the results are omitted.

\subsection{Structure of Control Frames and Active Receiver Management}

WDOS uses variants of the RTS/CTS handshake for channel probing. The frame formats of BRTS and modified CTS frames are shown in Fig. 3. The BRTS control frame contains two additional fields, A/D (Add/Drop) and Num. The A/D flag combined with the RA field manages a set of active receivers. When a sender has data frames to send to a currently dormant receiver, it activates the receiver by recording the receiver's address in the RA field and setting the A/D flag to 'Add'. Deactivation is more complex than activation and we develop two methods: an explicit and an implicit method. The explicit method records the address of deactivated node in the RA field and sets the A/D flag to 'Drop'. The explicit method is usually used to subdue receivers that wrongly think they are active. The implicit method uses the "More Data" flag specified in the IEEE 802.11 standard [3] to continue or stop the states of existing active stations. To deactivate, the sender notifies a receiver to switch to an inactive state by clearing the "More Date" flag. As a measure of fail-safe, the sender reconfirms the activeness of receivers by recording each of their addresses in a round robin manner. The Num field denotes the number of active receivers. The CTS control frame again is augmented with two fields, $\mathrm{R}$ and TA; R (Rate) specifies the desirable data rate and TA (Transmitter Address) is the address of the station transmitting the CTS frame.

\section{Analysis}

This section describes an analysis that derives the throughput of WDOS. To make the analysis simple, we make several assumptions. First, we assume that there are a fixed number of active receivers and there always are packets to send to each receiver. We assume each station can hear each other and there is no hidden terminal problem; anlaysis studies focus on the infrastructure mode. As shown in Section 3, WDOS repeats cycles which consist of up to four phases: the random backoff phase before BRTS, the BRTS transmission phase, the CTS resolution phase, and the frame transmission phase. Only the third phase, the CTS resolution phase, is different from the basic IEEE 802.11 standard from in terms of analysis. 


\subsection{Average CTS Resolution Time}

When an active station receives a BRTS frame, it determines the backoff period based on its relative channel quality. In case of a CTS collision, the channel probing mechanism repeats until a success. Recall that a channel quality p.d.f. is partitioned into $L$ intervals with the equal probability of $1 / L$, and each interval consists of $M$ slots. The probability that $k$ receivers transmit CTS frames in the $i^{\text {th }}$ interval, $P_{C T S}(i, k)$, is

$$
P_{C T S}(i, k)={ }_{N} \mathrm{C}_{k}\left(\frac{1}{L}\right)^{k}\left(1-\frac{i}{L}\right)^{N-k} \quad i \in(i, L), k \in(1, N) .
$$

Because the first CTS finishes the channel probing period, there should be no CTS transmissions before the $i^{t h}$ interval. Once a station selects an interval, it randomly chooses one integer from an interval $[0, \ldots, M-1]$. A collision occurs if two or more stations select the same interval and the same random number. Let $k$ be the number of stations that select the same interval. The conditional probability that a collision-free transmission of a CTS frame occurs at the $j^{\text {th }}$ slot, $P_{S}(j \mid k)$, is

$$
P_{S}(j \mid k)={ }_{k} \mathrm{C}_{1} \frac{1}{M}\left(1-\frac{j}{M}\right)^{k-1} \quad k \in(1, N), j \in(1, M) .
$$

The conditional probability of collision at the $j^{\text {th }}$ slot, $P_{C}(j \mid k)$, is

$$
P_{C}(j \mid k)=\left(1-\frac{j-1}{M}\right)^{k}-P_{S}(j \mid k)-\left(1-\frac{j}{M}\right)^{k} \quad k \in(1, N), j \in(1, M) .
$$

The probability that the first CTS is successfully transmitted in the $j^{\text {th }}$ slot of the $i^{t h}$ interval, $P_{S-C T S}(i, j)$, is given as

$$
P_{S-C T S}(i, j)=\sum_{k=1}^{N} P_{C T S}(i, k) P_{S}(j \mid k) \quad i \in(1, L), j \in(1, M) .
$$

Similarly, the probability that CTS frames collide in the $j^{\text {th }}$ slot of the $i^{\text {th }}$ interval, $P_{C-C T S}(i, j)$, is

$$
P_{C-C T S}(i, j)=\sum_{k=1}^{N} P_{C T S}(i, k) P_{C}(j \mid k) \quad i \in(1, L), j \in(1, M) .
$$

The average successful CTS resolution time, $\mathrm{E}\left[T_{S-C T S}\right]$, is given as

$$
\mathrm{E}\left[T_{S-C T S}\right]=\sum_{i=1}^{L} \sum_{j=0}^{M-1} P_{S-C T S}(i, j) *\left(((i-1) * M+j-1) * \sigma+T_{C T S}\right),
$$

where $T_{C T S}$ is the CTS transmission time and /sigma is the slot time. On the other hand, the average time of CTS collisions, $\mathrm{E}\left[T_{C-C T S}\right]$, is

$$
\mathrm{E}\left[T_{C-C T S}\right]=\sum_{i=1}^{L} \sum_{j=0}^{M-1} P_{C-C T S}(i, j) *\left(((i-1) * M+j-1) * \sigma+T_{C T S}\right) .
$$


Note that due to the variable data rate, the number of bytes transmitted during the same time duration varies. The probability that a receiver with the channel quality of the $i^{t h}$ interval is successfully selected, $P_{S-C T S}(i)$, is determined as

$$
P_{S-C T S}(i)=\sum_{j=0}^{M-1} P_{S-C T S}(i, j) .
$$

The average data size to be transmitted in a single cycle is represented as

$$
\mathrm{E}[D]=\sum_{i=1}^{L} D(i) * P_{S-C T S}(i)
$$

where $D(i)$ is the average data size transmitted in the $i^{\text {th }}$ interval. $D(i)$ varies depending on the channel conditions because a sender transmits data frames to each receiver for a fixed equal time duration in WDOS. $P_{S}(i)$ and $D(i)$ can not be decoupled because the value of $P_{S}(i)$ and the transmission rate are correlated. Equation (9), which considers the channel dependent throughput, reflects the multiuser diversity gains.

\subsection{Saturation Throughput of the Proposed Scheme}

Let $P_{t r}$ be the probability that there is more than one transmission attempt in a slot and $P_{S-B R T S}$ be the probability of a successful BRTS frame transmission. $T_{C-B R T S}$ denotes the time wasted for an unsuccessful BRTS transmission. Let $\mathrm{E}\left[T_{S}\right]$ and $\mathrm{E}\left[T_{C}\right]$ be the average time consumed for a successful and unsuccessful CTS transmission, respectively. Applying the same method used for the analysis of the basic IEEE 802.11 MAC [1], we express the saturated throughput of WDOS, $S$, as

$$
S=\frac{P_{S-B R T S} \mathrm{E}[D]}{\left(1-P_{t r}\right) \sigma / P_{t r}+\left(1-P_{S-B R T S}\right) T_{C-B R T S}+P_{S-B R T S}\left(\mathrm{E}\left[T_{S}\right]+\mathrm{E}\left[T_{C}\right]\right)} .
$$

Note that $P_{S-B R T S} \mathrm{E}[D]$ is the average size of successfully transmitted data frames in one cycle. The denominator of Equation (10) is the average cycle time. The first term is the average backoff time of the IEEE 802.11 DCF. The second term represents the average time wasted for each BRTS collision. $T_{C-B R T S}=$ $T_{B R T S}+D I F S$, where $T_{B R T S}$ is the transmission time for a BRTS frame.

The last term is the average time duration for the CTS resolution and data transmission in the case of a successful BRTS transmission. Even after a BRTS frame is transmitted successfully, WDOS suffers from CTS collisions. The last term, which consists of two parts $\mathrm{E}\left[T_{S}\right]$ and $\mathrm{E}\left[T_{C}\right]$, reflects this fact. $\mathrm{E}\left[T_{S}\right]$, the sum of the expected time of a successful CTS resolution and the expected time of a following data transmission, is expressed as $\mathrm{E}\left[T_{S}\right]=\mathrm{E}\left[T_{S-C T S}\right]+$ $\sum_{i} P_{S-C T S}(i) T_{S}(i)$, where $T_{S}(i)=T_{B R T S}+S I F S+T_{D}(i)+D I F S$ and $T_{D}(i)$ is the average time duration required to transmit frames in the $i^{\text {th }}$ interval. $\mathrm{E}\left[T_{C}\right]$, the expected time of an unsuccessful CTS resolution, is given as $\mathrm{E}\left[T_{C}\right]=$ 
$\mathrm{E}\left[T_{C-C T S}\right]+\sum_{i} \sum_{j} P_{C-C T S}(i, j) T_{C}$, where $T_{C}=T_{B R T S}+S I F S+D I F S$. For example, if $n$ frames can be transmitted in the $i^{\text {th }}$ interval, then $T_{D}(i)$ is given as $T_{D}(i)=n *\left(S I F S+H+T_{\text {payload }}(i)+S I F S+T_{A C K}\right)$, where $H$ and $T_{\text {payload }}(i)$ are the transmission time for the frame header and payload, respectively. $T_{A C K}$ is the ACK transmission time.

\section{Performance Evaluations}

We conducted analyses and ns-2 based [9] simulations to evaluate the performance of WDOS. The target system is the IEEE 802.11a which supports eight data rates from $6 \mathrm{Mbps}$ to $54 \mathrm{Mbps}[4]$. We limit the value of $B_{\max }$ to $\min (L * M-1,45) *$ SlotTime. The size of all data frames is fixed to 1500 bytes. Each simulation result is obtained from 20 repetitions, but the confidence intervals are omitted because they all are too small.

\section{$5.1 \quad$ Effect of $L$ and Multiuser Diversity}

We investigated the effects of the number of intervals, $L$, an important parameter that balances the trade-off relation between collisions and responsiveness, using the analytic model. We assume that all channels follow the Rayleigh fading model with the same average. To pin point the effect of $L$, eliminating the random effect, we assume $M=1$.

Two interesting aspects regarding the parameter $L$ are the effect of $N$ on the optimal values of $L$ and its sensitivity. Fig. 4 shows the optimal values of $L$ at different values of $N$. Because the optimal $L$ increases almost linearly as $N$ increases, we denote the optimal values of $L$ as $c N$, where $c$ is the slope of the graphs in Fig. 4. From this figure, $c$ is three or four. Now, let us examine the sensitivity of $L$. Fig. 5 shows the throughput as a function of $L$ for the case of $N=25$ when the average channel SNR is $-72 \mathrm{dBm}$. The throughput increases sharply as $L$ increases from 25 to 54 . Beyond $L=54$, the throughput increases rather slowly and the peak performance is obtained at $L=107$. The throughput decreases slowly as the number of intervals increases beyond $L=107$. From this graph, we can deduce that the throughput is not sensitive to $L$ when $L$ ranges from 54 to 150 . That is, if we let $L=c N$ and $c$ ranges from 2 to 6 , the performance of WDOS is not sensitive to the parameter $c$.

Fixing $c=4$ and $M=1$, we compared the analysis and simulation results to validate the analysis. Fig. 6 shows the throughput as a function of the number of active receivers when all the receivers have the same average channel quality of $-72 \mathrm{dBm}$ or $-75 \mathrm{dBm}$. Analysis and simulation results match well in all cases confirming the accuracy of the analysis. The multiuser diversity gains increase as the number of receivers increases. Note that the $3 \mathrm{~dB}$ difference of channel quality does not make the $3 \mathrm{~dB}$ throughput difference because of Medium Access Control (MAC) and physical layer overheads.

\subsection{Performance Comparison}

We compared the performance of WDOS to those of MAD and OSMA via computer simulations. For proper comparisons, we tuned up the MAD and OSMA 


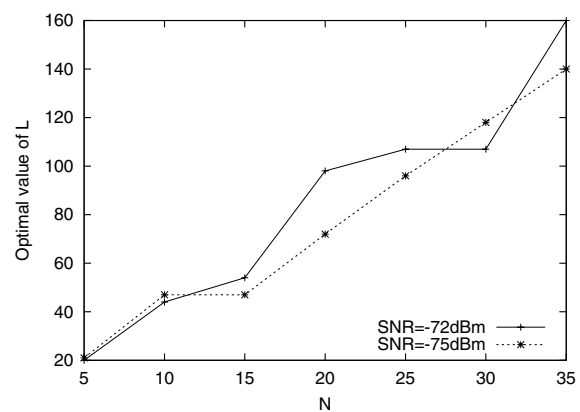

Fig. 4. Optimal values of $L$ at the different average channel qualities

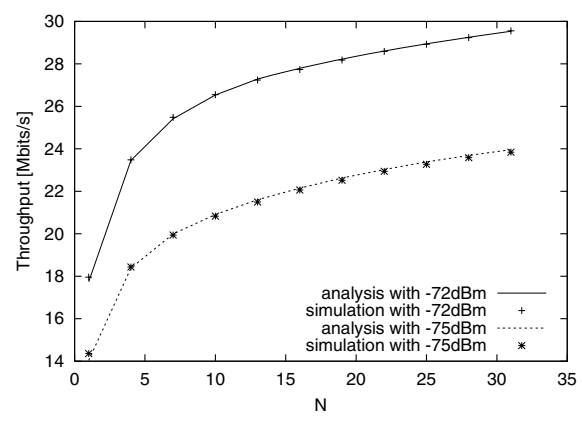

Fig. 6. Throughput: analytic vs. simulation results

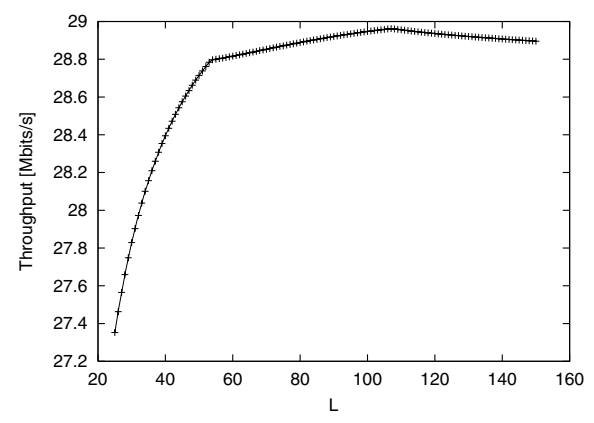

Fig. 5. Throughput with various $L$ when $N=25$ and $\mathrm{SNR}_{a v g}=-72 \mathrm{dBm}$

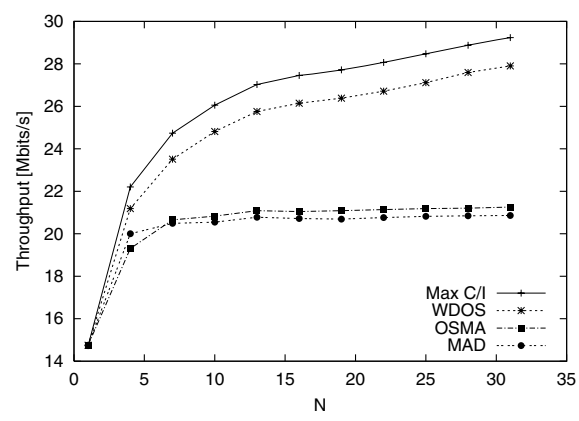

Fig. 7. Throughputs when the number of receivers increases from 1 to 31

protocols. MAD selects candidate receivers in a round-robin manner and chooses the final winner based on the proportional fair (PF) scheduling. In OSMA, the threshold of each receiver is set to a 70 percentage of its average channel quality. We also evaluated the performance of two algorithms, the max $\mathrm{C} / \mathrm{I}$ scheduler and the PF scheduler. We assume that these two schedulers know the channel conditions of all the receivers with the overhead of one BRTS/CTS exchange. The $\max \mathrm{C} / \mathrm{I}$ scheduler achieves the theoretical maximum throughput [8] while the proportional fair $(\mathrm{PF})$ scheduler provides temporal fairness [2]. We consider the Doppler shift [11] in evaluating the performance. In the case of long coherence time (typical indoor environments), WDOS may experience the performance degradation due to consecutive CTS collisions if $M=1$. To resolve these consecutive collisions, $M$ must be larger than one. In the following simulations, we fix $M=2$ and $c=3$.

Throughput Performance. In this suite of simulations, channels have i.i.d. Rayleigh fading with a $1 \mathrm{~Hz}$ Doppler shift while the average signal quality at each 


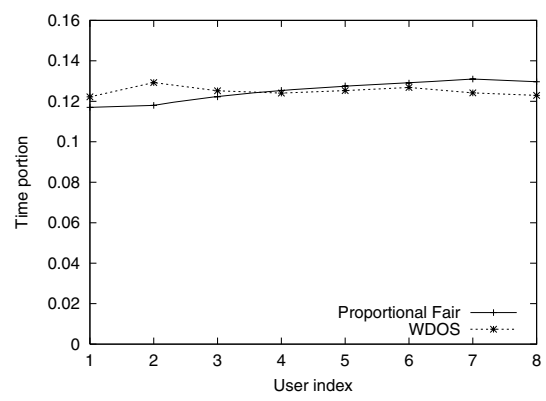

Fig. 8. Temporal fairness

receiver is $-72 \mathrm{dBm}$. Fig. 7 shows the throughput of WDOS, MAD, OSMA, and a max $\mathrm{C} / \mathrm{I}$ scheduler as a function of the number of receivers. The throughput of all the scheduling algorithms increases as $N$ increases from 1 to 5 . While the throughput of WDOS and the max C/I scheduler increases further beyond $N=$ 5 as $N$ increases, the throughput of MAD and OSMA saturates at around $N=$ 7. These results indicate that MAD and OSMA fail to fully realize multiuser diversity gains because they limit the candidate receivers. However, WDOS, which invites all receivers, fully achieves the multiuser diversity gains. When $N$ reaches to 30 , the throughput of WDOS is about $34 \%$ more than that of MAD and OSMA.

Temporal Fairness. In order to evaluate temporal fairness, we set up a simulation environment, where eight receivers have i.i.d. Rayleigh fading with a $4 \mathrm{~Hz}$ Doppler shift. Eight receivers have different average channel conditions; $-82 \mathrm{dBm}$ (user1), -81dBm (user2), $\cdots,-75 \mathrm{dBm}$ (user8). Fig. 8 depicts the time portions consumed by each user under PF scheduling and WDOS. Both scheduling algorithms assign the almost equal time-share (1/8) to each receiver. This reflects that the channel-aware backoff mechanism of WDOS guarantees the same access probability to all receivers even if their average channel conditions are different.

\section{Conclusions}

In this paper, we proposed a novel opportunistic scheduling algorithm called WLAN Distributed Opportunistic Scheduling (WDOS) for WLANs. In order to probe channel conditions, WDOS uses a modified RTS/CTS handshake, where each active receiver transmits a CTS frame after its own channel-aware backoff expires. Even if this channel-aware backoff mechanism invites all active receivers to report their channel conditions, the channel probing overhead is small since the backoff mechanism adopts a contention-based scheme. In addition, because the first respondent has the relatively best instantaneous channel quality among all the receivers, WDOS fully achieves multiuser diversity gains. Both analysis and simulation results indicate that WDOS outperforms MAD and OSMA. 
When the number of active receivers reaches to 30 , WDOS achieves up to $34 \%$ throughput improvement in the indoor environments. WDOS also has the property of temporal fairness; receivers evenly share the time resource regardless of different average channel conditions.

\section{Acknowledgements}

This work was, in part, supported by the Brain Korea 21 Project in 2006. This work was, in part, supported by grant No.R01-200400010-37202005 from the Korea Science \& Engineering Foundation. The ICT at Seoul National University provided research facilities for this study.

\section{References}

1. G. Bianchi, "Performance analysis of the IEEE 802.11 distributed coordination function," IEEE JSAC in Commun., vol.18, no.3, pp.535-547, Mar. 2000.

2. J. M. Holtzman, "CDMA forward link waterfilling power control," Proc. IEEE VTC 2000-Spring, Tokyo, Japan, pp.1663-1667, Jan. 2000.

3. IEEE Computer Society LAN/MAN Standards Committee, "Part 11: Wireless LAN Me-dium Access Control (MAC) and Physical Layer (PHY) Specifications," ANSI/IEEE Std 802.11, 1999 Edition.

4. IEEE Computer Society LAN/MAN Standards Committee, "Part 11: Wireless LAN Me-dium Access Control (MAC) and Physical Layer (PHY) specifications. High-speed Physi-cal Layer in the 5 GHz Band," IEEE Std 802.11a-1999(R2003). (Supplement to IEEE Std 802.11-1999).

5. A. Jalali, R. Padovani, and R. Pankaj, "Data Throughput of CDMA-HDR a High Effi-ciency-High Data Rate Personal Communication Wireless System," Proc. IEEE VTC 2000-Spring, Tokyo, Japan, pp.1854-1858, Jan. 2000.

6. Z. Ji, Y. Yang, J. Zhou, M. Takai, and R. Bagorodia, "Exploiting Medium Access Diversity in Rate Adaptive Wireless LANs," Proc. ACM MOBICOM 2004, Philadelphia, Pennsyl-vania, pp.345-359, Sep. 2004.

7. R. Knopp and P. Humblet, "Information capacity and power control in single cell multiuser communications," Proc. IEEE ICC 1995, Seattle, WA, pp.331-335, Jun. 1995.

8. A. Kogiantis, N. Joshi, and M. O. Sunay, "Effects of Scheduling on Transmit Diversity Performance in 1xEV-DV," Lucent Contribution to 3GPP2 TSG-C Standards Body, Dec. 2000.

9. NS-2, http://www.isi.edu/nsnam/ns, 2005.

10. Qualcomm, Inc., "1xEV: 1x Evolution IS-856 TIA/EIA Standard Airlink Overview," Nov. 7, 2001, Revision 7.2.

11. T. S. Rappaport, "Wireless Communications: principles and practice," 2nd Edition, Pren-tice Hall.

12. J. Wang, H. Zhai, and Y. Fang, "Opportunistic Packet Scheduling and Media Access Control for Wireless LANs and Multi-hop Ad Hoc Networks," Proc. IEEE WCNC 2004, Atlanta, Georgia, Mar. 2004. 